\title{
Erratum to: Extinction efficiencies of mixed aerosols measured by aerosol cavity ring down spectrometry
}

\author{
WANG Lei ${ }^{1,2}$, WANG WeiGang ${ }^{1} \&$ GE MaoFa ${ }^{2 *}$ \\ ${ }^{1}$ State Key Laboratory for Structural Chemistry of Unstable and Stable Species, Beijing National Laboratory for Molecular Sciences, \\ Institute of Chemistry, Chinese Academy of Sciences, Beijing 100190, China; \\ ${ }^{2}$ Beijing National Laboratory for Molecular Science, State Key Laboratory for Structural Chemistry of Unstable and Stable Species, \\ Peking University, Beijing 100871, China
}

\section{Erratum to: CHINESE SCIENCE BULLETIN, July 2012 Vol.57 No.20: 2567-2573 doi: 10.1007/s11434-012-5146-7}

In the original publication of this paper, the superscripted number relating to the current address of the corresponding author GE MaoFa has been documented incorrectly. The superscripted number should be "1" and not " 2 ". The corrected information should be presented as follows:

WANG Lei ${ }^{1,2}$, WANG WeiGang ${ }^{1} \&$ GE MaoFa ${ }^{1 *}$

${ }^{1}$ State Key Laboratory for Structural Chemistry of Unstable and Stable Species, Beijing National Laboratory for Molecular Sciences, Institute of Chemistry, Chinese Academy of Sciences, Beijing 100190, China;

${ }^{2}$ Beijing National Laboratory for Molecular Science, State Key Laboratory for Structural Chemistry of Unstable and Stable Species, Peking University, Beijing 100871, China

An online version of the original article can be found at http://www.springerlink.com/content/25kr83067347nlx0/?MUD=MP

Open Access This article is distributed under the terms of the Creative Commons Attribution License which permits any use, distribution, and reproduction in any medium, provided the original author(s) and source are credited. 